\title{
A new way home:
}

\section{Refugee young people and homelessness in Australia}

\author{
Jen Couch
}

Australian Catholic University

\begin{abstract}
This article gives voice to refugee young people experiencing homelessness. It is based on a project that conducted interviews with refugee young people and consultations with service providers. The research reveals that the profoundly under-recognised phenomenon of homelessness experienced by young people of refugee background is often hidden and does not match commonly held beliefs about homeless young people. The article examines the nexus between migration and homelessness in young people and how culture and ethnicity impact on young people accessing services. The stereotype of the homeless young person has been in place for many years and has dominated the research literature and the development of services. The study finds that this stereotype is alive and well in the perceptions of refugee young people and the agencies. Young people of refugee background often feel unable to access or attend these services. All the young people interviewed showed a capacity to negotiate new spaces of hope and belonging, despite dwelling in a 'landscape of exclusion' (Sibley 1995) - a testament to their strength, resilience and sense of agency.
\end{abstract}

Sibley, D. (1995), Geographies of Exclusion: Society and Difference in the West, London: Routledge.

Keywords: refugee, homelessness, youth, discrimination

This article gives voice to refugee young people experiencing homelessness. Whilst this article focuses on the voices of young people themselves, it is based on a broader research project that involved interviews with refugee young people, consultations with service providers and a review of available research and literature relating to refugee youth homelessness. The research revealed that the profoundly under-recognised phenomenon of homelessness experienced by young people of refugee background is often hidden and does not match commonly held beliefs about homeless young people. It also found that young people of refugee background often felt unable to access homelessness agencies. 


\section{Literature Review}

This cultural definition of homelessness has not only assisted academics to guide the translations of the various and complex dimensions of homelessness, but has also presented an adequate method of quantifying the number of people who are identified as being homeless in Australia as displayed in Chamberlain and Mackenzie's 2001 national homeless census (2003) and again in 2006 (2008). By contrast, Chamberlain and Mackenzie's reported statistics are consistent with the figures put forward by the Australian Institute of Health and Welfare on the Supported Accommodation Assistance Program Data Collection (2008) which, although only taking into account homeless people who use SAAP funded services, provides the welfare sector with another tool to estimate the homeless population and validates Chamberlain and Mackenzie's (2008) findings. Statistics such as these have been proactive in inciting the growing trend in the research and discussion surrounding the issues of homelessness. The increase of discussion has signified two things: one, that there is no denying that Australia is experiencing a growing homeless problem; and two, that there is a requirement of understanding the spectrum of homelessness. As a result there has been extensive qualitative and quantitative research into Australia's homeless population over the last decade with the purpose of understanding the extent of the nature of homelessness.

On any given night in Australia, an average of 104,000 Australians are identified as being homeless (Chamberlain \& Mackenzie, 1992) and recent data indicates that young people under 25 are in the majority (Australian Institute of Health and Welfare, 2010). Chamberlain and Mackenzie (ibid) have developed a three tier definition of homelessness:

Primary homelessness: Someone who is without acceptable accommodation, and has taken to 'sleeping rough', a term that denotes living on the street;

Secondary homelessness: Those who couch surf, temporarily staying with relatives or friends, in emergency accommodation or refuges;

Tertiary homelessness: Residents of private boarding houses who do not live within minimal housing standards (often sharing a communal bathroom or kitchen), without a fixed tenure.

There is limited research relating to homelessness experienced by refugee young people. In fact, the majority of literature demonstrates that whilst there has been much written on the subject of homelessness, there has been an over-representation of the experience of homeless men, with women's experiences not being captured (Austerberry \& Watson, 1986; Sharam, 2008), and the experiences of refugee young people being virtually invisible. The available literature recognises that the experiences of homeless refugee young people are similar to those of the broader population of homeless youth, but that refugee young people are at significant disadvantage. Their lives are further disrupted by issues relating to their refugee experience, re-settlement, lack of education as well as serious challenges to their adolescent development (Coventry, Guerra, Mackenzie \& Pinkey, 2002; Ransley \& Drummond, 2001; Refugee Young people and Housing Issues Working Group, 2002; Wallace, 1990).

In the year 2000, a total of 2067 young people entered Australia under the Humanitarian Program, indicating a rise of refugee young people arriving in Australia since 1991 (Coventry et al., 2002). The Centre for Multicultural Youth (2009) has since reported that in 2008/09, 66 per cent of Humanitarian Program arrivals to Australia have been under the age of 30 years. It is noted that this group of young people commonly enter Australia alone or with very little family or social networks and without certain accommodation (Refugee Young People and Housing Issues Working Group, 2002). 
Additional disadvantages that exacerbate refugee young people's vulnerability to homelessness include social isolation, economic hardship, racism and discrimination by real estate agents and employers, language barriers and cultural ignorance, family breakdown and poor mental health due to traumatic refugee experiences.

Despite limited data and information it has been estimated that between 500 and 800 refugee young people are homeless Australia wide, making the risk of homelessness for refugee young people six to ten times higher than Australian youth (Mackenzie cited in Coventry et al., 2002). It should be noted however, that these estimates are now over ten years old and given the significant numbers of young people who have entered Australian in this time, one can presume this estimate would now be much higher.

\section{Housing}

The experience of homelessness does not begin in Australia for a refugee young person. Many have been homeless in their country of origin (ASeTTS, 2008) and in Australia experience overcrowding in small properties (Ransley \& Drummond, 2001; ASeTTS, 2008).

Less than 10 per cent of refugee people successfully access public housing in the first 18 months of resettlement (Campbell cited in Coventry et al. 2002, p.51). According to the literature available housing options are frequently culturally inappropriate for a refugee young person (Coventry et al., 2002; Olliff, 2009) and many services working with refugee young people lack workers trained to work effectively with their circumstances and needs. (Hartley, 1990; Victorian Homeless Strategy, Ministerial Advisory Committee, 2001). Due to a lack of familiarity with services many refugee young people do not access emergency, transitional and public housing (Ransley \& Drummond, 2001; Refugee Young People and Housing Issues Working Group, 2002). In the same way refugee young people are also vulnerable to misinformation about social services and support networks (Ransley \& Drummond, 2001) leading to the under-representation of refugee young people in mainstream and culturally specific housing support services (Francis \& Cornfoot, 2007). Private rental is almost unattainable for refugee young people due to a lack of rental history, the lack of or a bad credit history (Victorian Homeless Strategy, Ministerial Advisory Committee, 2001; Francis \& Cornfoot, 2007) and the lack of finances for a bond, rent in advance and moving costs (Coventry et al., 2002). While there is assistance available for this purpose, many refugee young people are unaware or suspicious of these services. Another barrier to accessing the private rental market is racial discrimination by real estate agents and landlords (Kelly, 2004; Foley \& Beer, 2003; Coventry et al., 2002).

Many homeless refugee young people find couch surfing their only housing option (ASeTTS, 2008; Kelly, 2004). The Centre for Multicultural Youth (2010) and Beer and Foley (2003) reported that young people do not define their situation as being homeless when they move around sleeping between friends or families’ homes.

\section{Employment and Education}

Refugees have the lowest labour force participation rate among all immigrant groups due to a lack of formally recognised qualifications and work experience (Ransley \& Drummond, 2000), English language difficulties, poor levels of literacy in their first language, traumatic refugee experiences, disruption to their education and training, the different education system in Australia and a lack of confidence participating in the mainstream school settings (Ransley \& Drummond, 2000 and ASeTTS, 2008, Sharmin Zaman \& Degagu, 2002). It was also 
reported that refugee young people do not receive adequate assistance to get work placements or training and are discriminated against by employers (Olliff, 2009).

Refugee young people's employment prospects are further impeded by the decrease in entry-level positions and apprenticeship opportunities for young people in the workforce generally (Ransley \& Drummond, 2000). Those refugee young people who do secure employment are often employed in casual jobs and financially vulnerable as a consequence (ASeTTS, 2008). These difficulties in gaining suitable employment and ultimately a reasonable income contribute significantly to refugee young people's vulnerability to homelessness (Sharmin Zaman \& Degagu, 2002).

\section{Income}

Financial difficulties and insufficient available income lead refugee young people to be vulnerable to personal and car loans or hire purchases with high interest rates which often leads to large amounts of debt (Francis \& Cornfoot, 2007; ASeTTS 2008). Due to the high repayments, young people regularly fall into rent arrears, a situation that leads to eviction and ultimately to homelessness. Personal loans and fines make it impossible to clear a bad credit history, creating a further barrier to accessing the private rental market (ASeTTS, 2008). Many refugee young people are expected to send money to relatives in refugee camps overseas or to support family members unable to receive social benefits in Australia while struggling to pay their own rent, bills and other expenses on a Centrelink allowance (Coventry et al., 2002; Ransley \& Drummond, 2001).

\section{Physical and mental health}

Refugee young people have often experienced situations and conditions which have led to intense psychological distress such as imprisonment, torture, the witnessing of murder, separation from family members, sexual and physical assault and the deprivation of human rights. While many young people experience mental health problems during an experience of homelessness this is intensified for refugee young people by these past events in their lives (Ransley \& Drummond, 2001; Coventry et al., 2002). Refugee young people face the burden of these traumas at a vulnerable stage of adolescent development while simultaneously resettling in a new country (Coventry et al., 2002). Added to this, the experience of homelessness such as sleeping rough may bring back past experiences of being in a refugee camp or fleeing from war and lead to further depression and anxiety (ASeTTS, 2008).

An overwhelming amount of the literature reviewed identified poor mental health as a major contributor to young refugee homelessness. (ASeTTS, 2008; Ransley \& Drummond, 2000; Sharmin Zaman \& Degagu, 2002). In addition past experiences of sexual and physical abuse are a significant issue, particularly for women (ASeTTS, 2008; Dunbar, 1994; Coventry et al., 2002; Sharmin Zaman \& Degagu, 2002). Physical and sexual abuse postresettlement has also been noted within the context of family breakdown or conflict (Sharmin Zaman \& Degagu, 2002).

Also noted was the greater likelihood that this group of young people suffered from poor physical health. Conversely the use of illicit drugs among refugee young people was reported to be lower than in the broader population cohort (Coventry et al., 2002). 


\section{Family Conflict or Breakdown}

Family conflict or breakdown is identified as a common cause of refugee youth homelessness, particularly for accompanied young people who have resettled with their family or extended families (Stamenitis cited in Sharmin Zaman \& Degagu, 2002 and ASeTTS, 2008). Reasons for family conflict include cultural dislocation, intergenerational conflict, overcrowding, the remarriage of a parent, financial stress, an increase in family responsibility for the young person as well as a drug or alcohol addiction within the family (ASeTTS, 2008; Kelly, 2004; Refugee Young people and Housing Issues Working Group, 2002; Sharmin Zaman \& Degagu, 2002). Parents sometimes see their child's acquisition of the English language, western fashion or a new social life as an abandonment of traditional cultural beliefs and values leading to stress and conflict (Coventry et al., 2002).

Refugee young people often have additional family responsibilities. Many support their families by interpreting information relating to housing, income and employment issues and often need to accompany parents to appointments, impinging on the young person's time, social life and studies which can result in conflict (ASeTTS, 2008). Many other refugee young people enter Australia as unaccompanied minors and arrive in Australia without any family or extended family. With an absence of family support, these refugee young people are likely to be further disadvantaged and susceptible to homelessness (Coventry et al., 2002).

\section{Gender}

This lack of service access is further explored in relation to gender. It is noted that young women in particular are unlikely to initiate contact with mainstream services without advocacy and this may account for the under-representation of female refugee young people in the social security system (Gregory, 1993, cited in Ransley \& Drummond, 2001). Although gender is rarely a topic of discussion in literature, Coventry et al. (2002) have noted the increase of young women in the Humanitarian program. It is argued that the increasing isolation of these women is contributing to the risk of depression and mental health problems (Ransley \& Drummond 2001; Sharmin Zaman \& Degagu, 2002).

It is also noted that refugee young women need culturally sensitive support and information particularly in relation to early pregnancy, prenatal care, sexual violence, sexually transmitted disease and female genital mutilation. (Coventry et al., 2002). In addition Dunbar (1994) has suggested that as a result of the gendered vulnerabilities of refugees there is a need for further research to be conducted on the experiences and needs of refugee women in particular in relation to appropriate housing.

In summary, this review has drawn upon the body of available knowledge in relation to homelessness experienced by refugee young people. This literature is limited and outdated but the circumstances of refugee young people warrant further and contemporary research into the issues outlined here.

\section{At the Heart of the Story}

\section{Young Refugees speak about Homelessness}

Using a participatory research framework, this study aimed to provide a forum through which homeless refugee young people could share their stories in order to inform service provision. The data for this article was collected in 2010 as part of a joint research project between 
Australian Catholic University and the Centre for Multicultural Youth. Ethics clearance was obtained through the university.

The theoretical and methodological underpinnings of the research are derived from critical social theory (CST), intersectionality theory and narrative inquiry. From a critical perspective, knowledge is grounded in politics with the insistence that social phenomena be related to the historical whole, and to the structural context in which they are situated (Lather, 1991). Closely linked to, and compatible with, CST is intersectionality theory. Although the term was first articulated by Crenshaw in the 1990s, black feminist scholar, Patricia Hill Collins (1991) had previously written about the interlocking "matrices of domination" to describe the multiple and intersecting inequalities, derived from race, gender, class, and ability, that shape the lives of women (Crenshaw, 2000, para. 8). An intersectional analysis directs attention away from an exclusive focus on individual stories and experiences, to consideration of larger systemic and structural inequalities. By locating the issue of homelessness within a political and social context, critical theory can provide a practical and relevant framework for understanding the lived experiences of young people whilst simultaneously seeking strategies to challenge oppressive circumstances in their lives. Although narrative inquiry embraces many theoretical approaches, several assumptions are shared. One of these is a consensus as to the pervasive nature of stories and storytelling. Humans are storytelling beings who, individually and socially, lead storied lives. While narratives may take many forms, they generally consist of stories that include a chronological ordering of events and an effort to bring coherence to those events. Van Maanen (1988) spoke about "critical tales," or the use of narrative approaches within a critical framework. The interest in such tales is to shed light on larger social, political, symbolic, or economic issues. Thus, in telling stories about their experiences young people communicate not only their perceptions about what happened to them, but also the social, economic, and political meanings of those events.

This research was motivated by a strong commitment to exploring as fully as possible the experiences of refugee young people who have experienced homelessness. In order to spend sufficient time in building strong and trusting relationships with the research participants, a decision was made at the outset to limit the sample size. Each interview was separated by intervals of weeks or months, depending on the availability and readiness of the participants. Young people were recruited through reliable community and academic contacts who had well-established and trusting relationships with the young people. This was made possible by the researcher's long experience of working in the refugee and homelessness sectors. Information about the study was provided to potential participants by community members and workers. Young people who expressed interest contacted the researchers either by phone or e-mail at which time the study was described in more detail.

Because not all young people who have experienced homelessness have been associated with community organizations, the researcher also advertised the study in places young people frequent, such as local shopping strips and markets and community centers. All participants were given the choice of being interviewed alone or in a small group. The rationale for this option was the potential power of group interviews to provide a context in which individuals are able to analyse the struggles they have encountered and challenges they have faced, to simultaneously begin to collectivise their experiences, and develop a sense of empowerment as they began to see the possibilities for change. It was then the task of the researcher to analyse these references systematically within an "overall narrative frame" (Borland, 1991, p. 63). Conversations with young people were held several times during 2010 at local cafes, in a park, at emergency accommodation services and at friends' houses.

During face-to-face dialogic interviews, the researcher examined the various ways that homelessness is defined, experienced, and reproduced over time in different social 
contexts, and how, in turn, these experiences affect young people who are refugees. Consistent with the theoretical assumptions, one research goal is the development of knowledge in ways that have potential for emancipation and empowerment. A central aspect of the study design was, therefore, the use of dialogic and reflective techniques in which respondents became actively involved in the construction and validation of meaning (Maguire, 1987). An interview guide was used flexibly, with probes to encourage dialogue, critical reflection, and elaboration of responses.

Narrative interviewing requires an open-ended structure that allows the participants to direct the flow and focus of the conversation. While the researcher established the context for the interview, offering overall direction and providing affirming feedback, the participants took the lead, making associations among concepts as they understood these. Interviews were transcribed verbatim and analysed using techniques appropriate to the analysis of narrative data. While content and semantic analyses were drawn on to some extent (Becker, Beyene \& Ken, 2000), the main analytical technique was narrative analysis. This process involves breaking the narrative down into idea units in the interview transcript, which are numerically coded. Common themes were then determined from these units and collated. The product of the analysis is a "core narrative” (Garro, 2003) and accompanying themes.

The information presented in this section was provided by homeless refugee young people aged between 19 and 25 years, comprising five females and four males. These young people were homeless for between a few months and two years. At the time of interviews, some young people were 'couch surfing', two in emergency accommodation and others 'sleeping rough'. I also interviewed four young people who had transitioned out of homelessness. The young people interviewed originated from Sudan, Ethiopia, the Congo, and Liberia, Burma and Afghanistan.

The initial methodological challenge faced in this study was involving refugee young people in the research process. Many homeless refugee young people are somewhat invisible, not only in their new resettlement communities, but also within their particular refugee communities. Frequently, refugee young people may be considered 'hard-to-reach', and it often may seem easier to consult with leaders of refugee communities. However, as Zea and colleagues (2003) pointed out, “'hard-to-reach' refers not as much to objective characteristics of the target population, but rather to researchers' distance from that population” (p. 287). The first people one usually meets when venturing into refugee communities are refugee men and non-refugee service providers. It is much more difficult to connect with the more marginalised members of the community-particularly homeless young people.

The most essential part of the research approach rested on demonstrating to the young people that I wanted to hear about their problems so that I could help them figure out how to solve them. The research illustrates several important considerations when initiating and conducting valid ethical research with refugee young people. It takes an extensive time commitment in order to develop genuine relationships that are based on a commitment to contributing to the community. This ultimately leads to building trust. Another important issue to consider involves ensuring that refugee young people feel comfortable with the idea and process of being interviewed and with their interviewers. It was important to explain the purpose of what we were doing, and most importantly, to structure data collection in ways that were congruent with young people's cultures. The researcher became aware of the complexity involved in conducting competent research that is truly inclusive of homeless refugee young people who often are marginalised and under-represented members of their communities. These various methodological considerations highlight not only the difficulty of ensuring that young people's voices are heard during data collection, but also the importance of guaranteeing that they have the opportunities to genuinely represent themselves. 
What follows is a selection of key themes as identified by participating young people within these interviews

\section{Factors leading to homelessness}

The common causes for the young people to leave home were family conflict over parental rules or values, reconfigured families and overcrowding. Based on participants' descriptions of leaving home three could be classified as leaving voluntarily and six were asked to leave by their families.

A year after we got here my dad started going crazy. I didn't know it at the time. Nobody really did. He would disappear for a few hours and that turned into a couple of days. And then he got fired from his job. I quit school. I got a job and started trying to take care of my brothers. And it didn't work. Lost the house. Started moving.

My parents developed problems. My mum was experiencing women's freedom in Australia and my dad felt he was losing his family and it shook the whole family up.

The process of becoming homeless involved becoming increasingly alienated from the systems designed to support young people. Many struggled in school because of language difficulties, interrupted schooling and difficulties concentrating. Several identified as having learning difficulties, being alienated by peers and experiencing racism. They repeatedly described feelings of 'being different', of not belonging, of being outside and on the fringes. While they may not have had a clear picture of what they were excluded from, there was a strong perception that there was a larger community to which they didn't belong:

Was school hard? It wasn't hard as much as it was just not being able to concentrate on anything. Like I knew what I had to do and I knew I can't do it and all that but I didn't see any point. Because of everything, yeah.

The teachers didn't know how to teach me. They just didn't understand how to teach me. I have learning problems and they didn't understand how to get around them no matter how many times I explained it.

The impact of mental and emotional health on the experience of home and homelessness for these refugee young people was staggering and complex and beyond the scope of this report. However the interviews revealed how critical it is to acknowledge the devastating impact mental health problems have on young people resettling in Australia. Three of the young men interviewed are 'chronically homeless' and moving from place to place:

I move all the time it is normal for me.

Another young man was beaten in the Sudan and suffered a head injury. He also moved from place to place, poverty and mental illness contributing to his insecurity:

I live day to day. Some people have a plan. For me it is difficult because I do not know what will happen. 
A Somali young man lived in twelve places since his arrival in Australia five years earlier and at the time of the interview was living in a shelter in the city. He suffered from paranoia and during the interview illustrated his fear:

You look like an immigration officer. How many questions do you have left?...Sometimes I can't smile because I don't feel well. I'm not going to change.

All three young men were survivors of persecution and continually walk a fine line between being housed and living on the streets.

\section{Being homeless}

All the young people were in a perpetual state of moving. Some daily, others weekly or monthly, all said that the instability of their housing arrangements put them at risk.

Most young people found shelter by couch surfing. It was common for all young people to at some stage move from one friend's house to another. Some tried to access youth refuges, but said they did not feel comfortable. They thought of themselves as different from other homeless young people. Several had not thought of approaching a refuge because they were afraid:

I felt the most homeless in the refuge because I was with people who would use drugs and it felt dangerous.

Some young people said they preferred to avoid refuges because they were too rigidly regulated and intrusive:

Going to get help they force you to tell your story over and over again. It was horrible and does not make you feel welcome.

I looked after my whole family before I came to Australia. You can't take a kid like me and put him in a place where they are going to have rules galore because they are not going to stay. It needs to be a stable place. No one's going to mind having a place where they can come and eat and sleep and stuff like that but it can't be a place where you can't do anything.

Most of the participants did not want to live in accommodation where their daily activities were controlled. They wanted to live independently. Young people said they would have liked a facility with single-occupant rooms and common areas for cooking and leisure activities. Staff would be mentors and oversee the functioning of the facility and provide support to residents, respecting their autonomy and operating from a philosophy of empowerment.

Service providers who were perceived as not respecting the autonomy of young people were avoided unless absolutely necessary. Some young people chose to remain homeless instead of going into refuges in order to maintain their independence. All of the young people said they wanted to transition out of homelessness on their terms: maintaining autonomy was a critical issue for them. 
One homeless young person told his story of trying to find somewhere to live:

It was a nightmare. When I ran out of money. I went through the phone book, looking for names that were familiar to my country...I rang the Salvation Army. They told me there was no room and to ring back in a week. But I had no money and no place to stay.

Another young person found an Ethiopian restaurant soon after arrival and went there. The people in the restaurant rang a social worker they knew, otherwise the young person had to sleep in the restaurant.

None of the young people interviewed were able to find and maintain stable employment while being homeless and their lack of education was a major hurdle in finding a decent job:

I just feel stupid. I can't speak English. I have nowhere to live. I can't help my family because I can't even help me.

I got the job and I lost the job and then we lost the flat and back on the street. And basically that's the pattern I went through because it was quite obvious that I didn't have the maths and English skills or anything like that. Even the last couple of years I was in school, I wasn't fully there because of all the other things that happened. My life just went zigzag.

\section{Barriers to accessing housing}

All participants said they wanted secure shelter. Many had attempted this but were pulled back into homelessness because they were unable to secure adequate or stable housing. The process of securing shelter was complicated by the fact that many young people were unaware of support agencies or they found them hard to access:

I spent two hours on the train just going up and down trying to find where the service was. Its humiliating to feel you can't do anything and not be able to communicate.

When young people did seek out services, they often encountered barriers to access. Young people expressed concerns in relation to accessing services suitable to their culture, needs and age:

If there was a place especially for people like us who can't live at home I would go. But they reckon that they're all racist and full of junkies and it's much worse.

Generally the young people perceived that they were not liked, they noted that there were rarely youth workers from their communities at the agencies and when they left they did not feel supported.

Three young people who had spent time in emergency housing all noted that they thought agencies needed to do more follow up.

More preparation for ongoing life is needed. 
They felt that workers did not understand the lives of people like themselves or what they had been through. They wanted the agencies to learn about them - their backgrounds, culture, racism and the problems they experienced. However it did not matter if workers did not always understand, it was more important that they were friendly and supportive.

When asked about barriers to accessing housing and feeling at home in Australia all young people mentioned language. Without adequate English language and literacy it was hard for these young people to function in Australian society. A young man who lived in a rooming house said:

My English is a problem because I do not always understand the rules of renting a place and may get evicted because I do not understand the rules. Or sometimes notices are placed in the building for people to come together and I can't be involved because I can't read the sign.

Young people spoke of their experiences of racism when they were trying to access private rental. Several felt they were turned away because they were black and one young woman spoke about a youth worker ringing on her behalf to inspect a property but when the young woman herself arrived, the landlord told her it was rented.

Despite the many difficulties some young people had progressed out of homelessness.

\section{Facilitators to turning around homelessness}

All young people in this study who transitioned out of homelessness cited a trigger event that helped them decide it was time.

I had a baby and that saved me... I look back on him and say I am glad I got pregnant even though his dad and I broke up like a month after I got pregnant. And I look at him every day and know every day that's why I went and got some help.

One young person had a specific time they planned to exit homelessness:

When I turn eighteen, I'm going to start settling down and getting my life back together. Until then, I am probably going to keep myself lost.

After making the decision, all young people who began transitioning did so with the assistance of another person who helped them find stable housing and financial assistance. This was not always a housing or youth worker. One young person was 'adopted' by a friend's mum who provided him with a stable home, food and emotional support.

When faced with impending homelessness, a pregnant young woman spoke about going through the phone book looking for Sudanese names to call someone for financial help. Similarly another young woman who came unaccompanied to Australia spoke about other Liberians who helped her survive the first few months:

I met a Liberian on the train. She said, 'Call me if you need anything.' The first thing I said was, 'I don't know you and I hope one day I can give you something back, but right now I need some money for food.' She came that day with three bags of food: milk, cheese, meat and cereal. 


\section{Discussion}

The findings of this project are consistent with the earlier research. Homelessness is not a choice that these young people made freely or easily. Most young people perceived that they had no alternative to leaving home. These results suggest that homelessness is an outcome of a process whereby young people increasingly become disconnected from the support systems around them, such as family, school and community. The literature emphasised family reconfiguration and overcrowding as two primary causes of refugee youth homelessness, which our findings support. Some of the refugee young people interviewed for this research became homeless after experiencing school and community conflicts that lead to strained relations with family members.

This study confirmed that refugee young people face numerous barriers in their attempts to leave homelessness including lack of adequate income, education, job opportunities and language. A lack of long term appropriate shelter services were cited by young people as a major factor in their homelessness. Homeless refugee young people often had several episodes of homelessness before they were able to re-establish their lives. Effective assistance for these young people involves the development of a comprehensive, coordinated continuum of services from housing assistance to employment skills to community development delivered over an extended period of time.

Refugee young people moving to independent living often faced discrimination in the housing market. Some young people said they needed counselling, not just from their refugee experiences but also from the trauma of homelessness. When asked to identify values and characteristics a worker should have, young people expressed the primary importance of having compassion, a good heart and being accepting.

\section{What do you need? People who will keep you and give you hope for tomorrow.}

This research indicates that refugee young people experience significant barriers to accessing homelessness agencies. At present time, the feeling is very strong that there are barriers in place which are preventing homelessness agencies from providing information, services and programs to many young refugee people. Similarly there are barriers to do with migration, settlement, language and culture that are inhibiting young refugees accessing agencies. Evidence in support of these claims has been documented in the literature review and in the interviews. The fact that homelessness can cross all community boundaries is well known and accepted. However, it must be remembered that the way these problems are dealt with varies considerably not only between different communities but within each community. Failure to recognise this can lead to agencies adopting stereotypical responses that may miss the mark completely.

Not only did it appear that most young people had not accessed such services, it was disturbing to learn that many young people felt there was 'no place' for them within existing programs. This is a strong indication that existing services are not delivered in a way that is consistent with the needs of refugee young people. The young people interviewed were isolated and felt they were socially stigmatised which therefore excludes many accommodation options. Young refugees' expectations of services, as well as adequate service provision, the trial of new interventions and the provision of education and training to homelessness agencies, all require an immediate response. Not only do refugee young people not access services, they are absent from the literature. There must be more research to examine homelessness experienced by this group of young people. 


\section{References}

ASeTTS (2008). Hidden Homelessness the impact of homelessness on newly arrived youth. Perth, Australia: Association for Services to Torture and Trauma Survivors Inc.

Austerberry, H. \& Watson, S. (1986). Housing and homelessness: a feminist perspective. London: Routledge \& Kegan Paul.

Australian Institute of Health and Welfare (2010). Government-funded specialist

homelessness services SAAP National Data Collection annual report 2008-09. Retrieved August 20, 2010 from http://www.aihw.gov.au/publications/hou/219/11235.pdf.

Becker, G., Beyene, Y. \& Ken, P. (2000). Health, welfare reform, and narratives of uncertainty among Cambodian refugees. Culture, Medicine and Psychiatry, 24, 139163.

Borland, K. (1991). “That's not what I said”: Interpretive conflict in oral narrative research. In S. B. Gluck \& D. Patai (Eds.), Women's words: The feminist practice of oral history (pp. 63-75). New York:Routledge.

Chamberlain, C., Johnson, G. \& Theobald, J. (2007). Homelessness in Melbourne: Confronting the Challenge. Melbourne, Australia: Center for Applied Social Research RMIT University.

Chamberlain, C. \& Mackenzie, D . (2008). Counting the homeless, 2006. Canberra, Australia:Australian Bureau of Statistics..

Collins, P. H. (1991). Black feminist thought: Knowledge, consciousness, and the politics of empowerment. New York:Routledge.

Council to Homeless Persons (2009). Fact Sheet Youth Homelessness in Victoria. Retrieved August 13, 2010 from http://www.chp.org.au/public_library/ youth_homelessness. chtml

Coventry, L., Guerra, C., Mackenzie, D. \& Pinkney, S. (2002). Wealth of All Nations Identification of strategies to assist refugee young people in transition to independence - a report to the National Youth Affairs Research Scheme. Hobart, Australia:Australian Clearing House for Youth Studies.

Crenshaw, K. (2000). The intersectionality of race and gender discrimination. Retrieved August 202010 from http://www.isiswomen.org/womenet/apgr-list/archive/ msg00013.html

Francis, S. \& Cornfoot, S. (2007). Working with multicultural youth: Programs, Strategies and Future Directions. Canberra, Australia:Australian Research Alliance for Children and Youth.

Garro, L. C. (2003). Narrating troubling experiences. Transcultural Psychiatry, 40(1), 5-43.

Hartley, R. (1990) Homelessness Still a Problem. Family Matters, 27, pp.27-28.

Ivan-Smith, E. \& Johnson, V. (1998). The Way Forward. Lines of communication and power. In V. Johnson, E. Ivan-Smith, G. Gorgon, P. Pridmore \& P. Scott (Eds.) Stepping Forward: Children and Young People's Participation in the Development Process, (pp. 291-299). London:Intermediate Technology Publications.

Lather, P. (1991). Getting smart: Feminist research and pedagogy with/in the postmodern. New York:Routledge, Chapman, \& Hall.

Maguire, P. (1987). Doing participatory research: A feminist approach. Amherst, MA:University of Massachusettes, Center for International Education.

Olliff, L. (2009). Amplifying the Voices of Young Refugees. Refugee Council of Australia.

Ransley, C. \& Drummond, S. (2001). Homeless Twice: Refugee Young People and Homelessness in Victoria. Carlton, Victoria: Centre for Multicultural Youth Issues. 
Refugee Young People and Housing Issues Working Group (2002). Culturally Appropriate Housing Options. Victoria: Victorian Settlement Planning Committee.

Said, E.W. (2001). Reflections on Exile and Other Literary and Cultural Essays. London:Granta Books.

Sharam, A.(2008). Going it Alone: Single, Low Needs Women and Hidden Homelessness. Brunswick:Women's Information, Support and Housing in the North.

Sharmin Zaman, R. \& Degagu, Y. (2002). We Should Not Be Homeless, A study of needs and social networks of culturally diverse, homeless young people in the City of Maribyrnong. West Footscray, Australia: Footscray Youth Housing Group.

Silbey, D. (1995) Geographies of exclusion: Society and difference in the West. London:Routledge.

Zea, M.C., Reisen, C.A., \& Diaz, R.M. (2003). Methodological issues in research on sexual behavior with Latino gay and bisexual men. American Journal of Community Psychology, 31, 281-291.

\section{Biographical Note}

Jen Couch teaches youth work at the Australian Catholic University. Prior to joining ACU she worked for over twenty years in the community sector in Australian and India. Her work has focused on highly marginalised young people in the areas of refugee settlement, rights and participation, torture and trauma and capacity building. 